OPEN ACCESS

Edited by:

Peng Zhang,

Vanderbilt University, United States

Reviewed by:

Piotr Soja,

Kraków University of Economics,

Poland

Xuedong Liang,

Sichuan University, China

*Correspondence:

Erik Westphal

erik.westphal@uni-rostock.de

Specialty section:

This article was submitted to

Blockchain Technologies,

a section of the journal

Frontiers in Blockchain

Received: 28 June 2021 Accepted: 16 August 2021

Published: 26 August 2021

Citation:

Westphal E and Seitz H (2021) Digital and Decentralized Management of

Patient Data in Healthcare Using

Blockchain Implementations.

Front. Blockchain 4:732112.

doi: 10.3389/fbloc.2021.732112

\section{Digital and Decentralized Management of Patient Data in Healthcare Using Blockchain Implementations}

\author{
Erik Westphal $^{1 *}$ and Hermann Seitz ${ }^{1,2}$ \\ ${ }^{1}$ Chair of Microfluidics, Faculty of Mechanical Engineering and Marine Technology, University of Rostock, Rostock, Germany, \\ ${ }^{2}$ Department Life, Light \& Matter, Faculty of Interdisciplinary Research, University of Rostock, Rostock, Germany
}

Blockchain solutions offer efficient approaches for trustworthy data management, especially in the medical field when storing and processing sensitive patient data. Many institutional and industrial facilities have already recognized the importance of the technology for the health sector and have also formulated basic ideas, concepts and main use cases, but concrete implementations and executions are comparatively rare. This mini review examines current research on specific blockchain implementations in healthcare that go beyond the state of concept studies or theoretical implementation ideas and describes the most promising systems based on systematic literature research. The review shows that secure storage and easy access to complete patient data is becoming increasingly important. Blockchain technology can be used as a secure, transparent and digital way to meet these needs. Hybrid solutions consisting of conventional data storage and blockchain-based access management are increasingly being developed and implemented. The automation of blockchain processes through smart contracts is also recommended. The review further reveals ambiguities in the use of permissioned and permissionless blockchain frameworks, machine learning $(\mathrm{ML})$ integration as well as the question of which data should be stored in the blockchain and how this should be viewed legally. Therefore, there is still a need for further research, especially on these aspects, in order to further establish the use of blockchains in healthcare.

Keywords: medical blockchain, digital healthcare, patient data management, electronic medical records, decentralized secure storage, health asset tracking

\section{INTRODUCTION}

In the course of their lives, patients generate data that are stored in central databases as a result of various events at different facilities or via different software structures (Azaria et al., 2016; Chen et al., 2018). The responsibility for the data usually lies with the respective operator of the database and not with the patient, which makes easy access to all data and control over the transfer and use of personal data almost impossible for them (Riso et al., 2017; Ballantyne, 2020). With the current widespread management of medical data, there is no guarantee of the integrity or reliability of patient records and the risk of data loss or data misuse is great (Chen et al., 2018; Lv and Piccialli, 2021). What is known as blockchain technology offers a promising new framework to enable and support the digital, secure and reliable integration of health information across various applications and stakeholders 
(Riso et al., 2017; Chen et al., 2018; Qiu et al., 2018; Kassab et al., 2019; Vyas et al., 2020). By setting up a seamless, decentralized data platform, for example information on medical records, records of authorization and proof of utilization of the data, provider directories, information on medicines and their supply chains as well as insurance and damage information can be recorded, tracked and managed securely and digitally (Agbo et al., 2019; Narikimilli et al., 2020). In the process, the blockchain is essentially a distributed database structure for the medical data in which all transactions or digital events are checked and stored by all parties participating in the database (Crosby et al., 2016; Huang and Foysal, 2021; Wu et al., 2021). This decentralization of the data as well as the higher security and transparency in data processing improve interoperability, quality and cooperation in the healthcare system enormously, reduce administrative costs and increase the authenticity and verifiability of the information (Azaria et al., 2016; Narikimilli et al., 2020).

A systematic literature research is chosen as the methodology for this study. Previous research on the topic, in our case blockchain for healthcare, is identified and analysed regarding to a specific research question. In this mini review, we identified and examined the following research question: What are the important and specifically implemented as well as programmed implementations of blockchain technology in healthcare that go beyond the state of concept studies or theoretical implementation ideas?

In order to find scientific publications on this research question, a keyword search was carried out in the scientific databases of Google Scholar, IEEE Xplore and ACM Digital Library. To identify relevant papers, the keywords "blockchain healthcare," "blockchain medical storage," "blockchain electronic medical records" and "blockchain healthcare supply chain" were used for the search queries. The times of the publications were then sorted by date and only publications from 2016 onwards were considered. Appropriate papers were then selected by reading the publications.

In the next section, current major blockchain implementations in the healthcare sector are examined in detail. For this purpose, there is a classification into three relevant main use cases of blockchain implementations in healthcare, based on categorizations from previously published reviews (Agbo et al., 2019; Narikimilli et al., 2020). The main use cases are the secure storage of medical records, the electronic medical data access as well as the associated permission management and the supply chain and health asset tracking. This is followed by a critical discussion of the research results and a conclusion in which a summary of the work and future development priorities are shown.

\section{BLOCKCHAIN IMPLEMENTATIONS IN HEALTHCARE}

\section{Secure Storage for Medical Records}

Accurate and complete patient data is a valuable asset for patients and clinicians who may be performing further treatments (Chen et al., 2018). Secure storage with easy accessibility and the protection of the privacy of medical data are therefore important issues. Usman and Qamar (2020) have developed a blockchain-based system for storing highly sensitive and private patient data with which these health data can be efficiently stored and securely distributed. The solution also ensures the data protection of medical records through the use of a permissioned blockchain based on the Hyperledger platform (Cachin, 2016). This system also allows patients to play an active role in managing their medical records and to be in control of who can add new records and view their medical history (Usman and Qamar, 2020).

However, with the increasing use of electronic solutions for processing medical information, the amount of data to be processed is also increasing rapidly (Li et al., 2018). The medical data can easily be stolen, manipulated or deleted while they are being stored and processed, which is why Li et al. (2018) have developed a novel blockchain-based data storage system for relatively large amounts of data. With the system, users can permanently save important data and continuously check for authenticity. During the treatment process, patient data in this study is created in text and multimedia formats, then subsequently protected against unauthorized access and stored in encrypted form. This is done using various cryptographic algorithms and file storage schemes as well as an identity information system in which the user identities are not linked to the medical data. The storage takes place in the form of transactions that are transmitted to the blockchain network and collected and stored in blocks of a defined data size. If the data in the database is then manipulated or damaged, the data information can be accessed and checked via the blockchain (Li et al., 2018).

The healthcare industry already manages personal patient data, diagnostic reports and medical prescriptions or reports in an electronically centralized manner (Kumar et al., 2020). However, with this it is difficult to protect the privacy of the patient, which is why Kumar et al. (2020) developed a blockchainbased system for storing medical data with distributed off-chain data storage via the Interplanetary File System (IPFS) (Protocol Labs Inc., 2021). For this purpose, a consortium blockchain (permissioned) framework with a private identity-based access control for the storage of special data information is implemented in the study. Furthermore, an IPFS off-chain storage model is implemented to manage the original patient data, since in the blockchain, according to Kumar et al. (2020), only a cryptographic hash value (Pilkington, 2016) of the patient information is stored due to the scalability.

Zhou et al. (2018) have developed a blockchain-based storage system for medical health insurance data that is tamper-proof and offers users a high level of credibility for their data. This system is designed to help insurance companies track and receive relevant medical information about patients both rapidly and easily (Zhou et al., 2018).

\section{Electronic Medical Data Access and Permission Management}

Before, the secure storage of medical records was still about the secure, digital storage and processing as well as the distribution of mostly incoherent patient data (e.g., diagnoses by a specialist for 
different patients), it is now more about the storage, processing and, in particular, accessibility of temporal and patient-specific related data in the form of an electronic medical record.

The use of medical patient files for various purposes always requires the consent of the patients, who currently grant or refuse these mostly in paper form and then no longer have any control or overview over their files (Tith et al., 2020). Tith et al. (2020) have developed a system with which patients can flexibly manage their consents and health organizations can also efficiently obtain and manage these consents for various purposes. The electronic system uses a dedicated access control based on a permissioned blockchain with Hyperledger implementation. In this study, all metadata of patient records, consents and data access are immutably stored in the blockchain and shared by the participating parties. In addition, a program code based on smart contracts or rather chaincode (Cachin, 2016) was created, which according to Tith et al. (2020) automates the business logic for managing patient consent.

However, strict regulation and bureaucratic inefficiency have greatly slowed the development of electronic medical records in the past (Azaria et al., 2016). Driven by new developments in electronic information technology, electronic records are now enjoying increasing acceptance (Fan et al., 2018). New technical innovations as well as an increased interest of patients in their personal data caused Azaria et al. (2016) to develop a new, decentralized data management system for the treatment of patient data based on blockchain technology. The system provides patients with a comprehensive, unchangeable log and easy access to their medical data, regardless of the facility and software system. The decentralized data system of these study manages authentication, trustworthiness, accountability and data exchange through the use of characteristic blockchain mechanisms such as smart contracts (Christidis and Devetsikiotis, 2016), mining (Christidis and Devetsikiotis, 2016) and proof of work (Gervais et al., 2016). In addition, it can be integrated into existing local data storage solutions and enables a data economy between patients, researchers and authorities (Azaria et al., 2016).

Generally, digital data for the same patient is collected and stored in different hospitals (Fan et al., 2018). However, for security and data protection reasons it is difficult to summarize or exchange the data between the institutions, which is why Fan et al. (2018) developed a blockchain-based information management system that cryptographically processes patient information and enables a secure exchange of information. With this system, extensive datasets can also be securely managed and patients, doctors, researchers and other third parties can easily have secure access to local databases of various institutions (Fan et al., 2018).

Healthcare management encompasses many different processes (e.g., managing finances, patients, legal issues etc.) and medical workflows related to patient care (Khatoon, 2020). Khatoon (2020) has developed a management system based on smart contracts for the intelligent and digital management of medical data in the healthcare sector and for the optimization of complex medical processes. During the implementation, representations of real medical files are created and various data of medical work processes are stored de-centrally in a blockchain. Access rights to metadata, authorization management and authorization rules for data access are then defined via smart contracts. The information is cryptographically secured and stored in a special, compressed form in a blockchain block (Khatoon, 2020).

\section{Supply Chain and Health Asset Tracking}

Healthcare supply chains have a significant impact on patient safety and the quality of medical treatments (Jayaraman et al., 2014). Effective supply chain management is crucial in healthcare in order to build the best possible procurement, ordering, forecasting and sales system, as well as to reduce costs and improve patient outcomes (Omar et al., 2021). For this reason Omar et al. (2021) have developed a blockchain-based system built on smart contracts and a decentralized storage system that improves the contract process for supply chains in the healthcare system. In this process, manufacturers, sellers, buyers and healthcare providers are connected to one another via a decentralized Ethereum (Buterin, 2014) network. Transparency, data origin and data immutability are already defined in the contract process and used for the implementation of automated procurement processes (Omar et al., 2021).

Blockchain technology has the potential to handle and track supply chain processes in healthcare and other areas very efficiently (Abbas et al., 2020). Abbas et al. (2020) have developed a novel management and recommendation system for drug supply chains based on blockchain and machine learning (ML) (Beam and Kohane, 2018). The system consists of a permissioned Blockchain, through which the drug supply chain is continuously monitored and authentically tracked, as well as an ML module that has been trained with a drug review dataset to recommend the bestrated drugs (Abbas et al., 2020). The ML module is also integrated into the blockchain via a Representational State Transfer Application Programming Interface (RESTful API) (Maleshkova et al., 2010).

Supply chains are complex structures that span multiple organizational and geographical boundaries and represent a critical infrastructure for medical services, especially in the healthcare sector (Musamih et al., 2021). For this, an efficient traceability of products in supply chains in the healthcare sector based on a permissionless blockchain was developed by Musamih et al. (2021). With this solution, products are automatically tracked via smart contracts and decentralized off-chain storage, intermediaries are superfluous and the transaction history is unchangeably and securely documented (Musamih et al., 2021).

In order to track, identify, communicate with and localize products and product data, radio-frequency identification (RFID) systems are increasingly being used in the healthcare sector (Figueroa et al., 2019). Figueroa et al. (2019) have developed a product-based access control system for RFID systems that executes access guidelines from a decentralized application based on a permissionless blockchain. With this implementation, for example, surgical instruments can be equipped with an RFID tag and their use and location can be tracked (Figueroa et al., 2019). 
TABLE 1 | Examined publications with special applications of blockchain implementations, the framework used and example applications.

\begin{tabular}{|c|c|c|}
\hline Use case & Framework & Example applications \\
\hline \multirow[t]{2}{*}{ Secure storage for medical records } & Permissioned blockchain & Usman and Qamar (2020), Kumar et al. (2020) \\
\hline & Permissionless blockchain & Li et al. (2018), Zhou et al. (2018) \\
\hline \multirow[t]{2}{*}{ Electronic medical data access and permission management } & Permissioned blockchain & Tith et al. (2020) \\
\hline & Permissionless blockchain & Azaria et al. (2016), Fan et al. (2018), Khatoon (2020) \\
\hline \multirow[t]{2}{*}{ Supply chain and health asset tracking } & Permissioned blockchain & Abbas et al. (2020) \\
\hline & Permissionless blockchain & Omar et al. (2021), Musamih et al. (2021), Figueroa et al. (2019) \\
\hline
\end{tabular}

\section{DISCUSSION}

It has already been recognized in a previous review (Agbo et al., 2019) that there is a lack of adequate prototype blockchain implementations. Therefore, this study aims to consider different blockchain implementations for a digital and decentralized management of patient-specific health data. Table $\mathbf{1}$ gives an overview of the implementations of various use cases, the blockchain framework used and the example applications.

The research found that secure storage and easy access to complete patient data across different facilities and systems is becoming increasingly important. In particular, the development of an electronic medical record is discussed in many publications and linked to the principles of blockchain technology. With this, a system of blockchain-based information and transaction storage as well as database-based storage of medical patient data is often implemented, which is a new methodology compared to those described in previous reviews (Agbo et al., 2019; Narikimilli et al., 2020). Blockchain mechanisms are used, among other things, to store aggregated information on as well as location information to more extensive diagnostic and multimedia files securely, transparently and digitally. More extensive data is usually stored in conventional database or cloud storage and secured by special data storage schemes or in additional blockchain modules to be integrated. In contrast to solutions reported in a previous review (Agbo et al., 2019), the latest implementation solutions use specially developed decentralized file storage systems to store larger amounts of data. Essentially, however, it can be stated that storing larger amounts of data in a blockchain does not make sense due to high storage costs and validation times. In addition, it is often not specifically described which information and data is stored in what form and to what extent in the blockchain. Research and development into authentic tracking of healthcare supply chains has also increased significantly in recent years. There, smart contracts in connection with blockchain implementations for the automated handling of contract, procurement and documentation processes are central elements. The integration of additional hardware for data acquisition such as RFID tags and the use of ML for data analysis, which was not covered in prior reviews (Agbo et al., 2019; Narikimilli et al., 2020), also enable decentralized and transparent visualization and storage of real-time data. It is also possible to extract relevant findings from large amounts of data using ML algorithms, which means that only the analysis results instead of the entire analysis data have to be stored in the blockchain. However, aspects of data protection and the accessibility of information via specific blockchain frameworks must be considered. Practitioners should therefor focus more on the further development of hybrid solutions from conventional data storage and secure, blockchain-based access control. There are already solutions here, but these are still at a very early stage of development. Users should always work out exactly whether the solutions should be implemented in the form of a permissioned or permissionless blockchain. In addition, customer aspects must be included in the development work. Moreover, existing implementations of blockchain solutions for the traceability of medical supply chains are to be further developed so that automated smart contracts control the processes and, for example, create authorizations, process data and document transactions. Researchers, on the other hand, should focus more on the effective use of blockchains in combination with $\mathrm{ML}$ and on investigating the legal basis for data storage in the specific blockchain frameworks. Research in these areas is still very inadequate.

\section{CONCLUSION}

Current research on implementations of blockchain technology in healthcare that go beyond the state of concept studies or theoretical implementation ideas was explored in this mini review. Also, major publications were examined in detail and categorized into three different main use cases of blockchain implementations in healthcare. The study shows, that secure storage and access to medical data are becoming increasingly important. The development of a secure, reliable and digital solution for this need is increasingly linked to the principles of blockchain technology. It is shown that hybrid solutions based on conventional data storage and secure, blockchain-based access control are more effective and economical than complete data storage in a blockchain. In addition, automated blockchain solutions based on smart contracts are recommended, e.g., when tracking medical supply chains.

In the future, specific guidelines for the electronic storage of patient data, medical products, devices and materials used etc. should be developed, which are important for patients and indispensable for any further treatment. Then the information should be defined that can be encrypted but stored publicly via permissionless blockchains as well as the information that is encrypted and in addition may only be accessible to authorized network participants in permissioned blockchains. With this, the storage of larger amounts of data must also be considered. Further studies with decentralized off-chain storage systems such as IPFS are conceivable for that as well as studies with ML methods for extracting and storing only relevant information from large 
amounts of data. The securing of the anonymity of the patient data to be stored de-centrally, the legal framework and the protection against data theft by criminal parties must also be considered. By then, at the latest, prototypical applications must increasingly be developed and established that go beyond early stage implementations.

\section{AUTHOR CONTRIBUTIONS}

EW conceived the research idea and provided a first draft of the manuscript. HS supported and supervised the process and revised

\section{REFERENCES}

Abbas, K., Afaq, M., Ahmed Khan, T., and Song, W.-C. (2020). A Blockchain and Machine Learning-Based Drug Supply Chain Management and Recommendation System for Smart Pharmaceutical Industry. Electronics 9, 852. doi:10.3390/ electronics 9050852

Agbo, C., Mahmoud, Q., and Eklund, J. (2019). Blockchain Technology in Healthcare: A Systematic Review. Healthcare 7, 56. doi:10.3390/ healthcare7020056

Azaria, A., Ekblaw, A., Vieira, T., and Lippman, A. (2016). "MedRec: Using Blockchain for Medical Data Access and Permission Management," in 2nd International Conference on Open and Big Data (OBD) (IEEE), Vienna, Austria, August 22-24, 2016.

Ballantyne, A. (2020). How Should We Think about Clinical Data Ownership? J. Med. Ethics 46, 289-294. doi:10.1136/medethics-2018105340

Beam, A. L., and Kohane, I. S. (2018). Big Data and Machine Learning in Health Care. JAMA 319, 1317-1318. doi:10.1001/jama.2017.18391

Buterin, V. (2014). A Next Generation Smart Contract and Decentralized Application Plattform. White Paper. Available at: https://ethereum.org

Cachin, C. (2016). Architecture of the Hyperledger Blockchain Fabric. Zurich: IBM Research.

Chen, Y., Ding, S., Xu, Z., Zheng, H., and Yang, S. (2018). Blockchain-Based Medical Records Secure Storage and Medical Service Framework. J. Med. Syst. 43, 5. doi:10.1007/s10916-018-1121-4

Christidis, K., and Devetsikiotis, M. (2016). Blockchains and Smart Contracts for the Internet of Things. IEEE Access 4, 2292-2303. doi:10.1109/ ACCESS.2016.2566339

Crosby, M., Nachiappan, N., Pattanayak, P., Verma, S., and Kalyanaraman, V. (2016). "BlockChain Technology: Beyond Bitcoin," in Applied Innovation Review (2016), 6-19. Berkeley, USA: Applied Innovation Review.

Fan, K., Wang, S., Ren, Y., Li, H., and Yang, Y. (2018). MedBlock: Efficient and Secure Medical Data Sharing via Blockchain. J. Med. Syst. 42, 136. doi:10.1007/s10916-018-0993-7

Figueroa, S., Añorga, J., and Arrizabalaga, S. (2019). An Attribute-Based Access Control Model in RFID Systems Based on Blockchain Decentralized Applications for Healthcare Environments. Computers 8, 57. doi:10.3390/computers8030057

Gervais, A., Karame, G. O., Wüst, K., Glykantzis, V., Ritzdorf, H., and Capkun, S. (2016). "On the Security and Performance of Proof of Work Blockchains," in Proceedings of the 2016 ACM SIGSAC Conference on Computer and Communications Security, Vienna, Austria, October 24-28, 2016 (New York, NY, USA: Association for Computing Machinery), 3-16.

Huang, G., and Foysal, A. A. (2021). Blockchain in Healthcare. TI 12, 168-181. doi:10.4236/ti.2021.123010

Jayaraman, R., Taha, K., Park, K. S., and Lee, J. (2014). "Impacts and Role of Group Purchasing Organization in Healthcare Supply Chain," in Proceedings of the 2014 Industrial and Systems Engineering Research Conference, Montréal, Canada, 31 May-3 June 2014. the first draft. EW and HS worked together to revise and fine-tune the final version of the manuscript. Both authors contributed to the article and approved the submitted version.

\section{FUNDING}

This research was funded by the European Union, which was made available through the European Regional Development Fund (ERDF) and the Ministry for Economics, Employment and Health of Mecklenburg-Vorpommern, Germany, grant number TBI-V-1-345-VBW-118.

Kassab, M., DeFranco, J., Malas, T., Destefanis, G., and Graciano Neto, V. V. (2019). "Investigating Quality Requirements for Blockchain-Based Healthcare Systems,” in 2019 IEEE/ACM 2nd International Workshop on Emerging Trends in Software Engineering for Blockchain (WETSEB) (IEEE), Montréal, Canada, 27 May 2019, 52-55.

Khatoon, A. (2020). A Blockchain-Based Smart Contract System for Healthcare Management. Electronics 9, 94. doi:10.3390/ electronics9010094

Kumar, R., Marchang, N., and Tripathi, R. (2020). “Distributed Off-Chain Storage of Patient Diagnostic Reports in Healthcare System Using IPFS and Blockchain," in 2020 the 12th International Conference on Communication Systems Networks (COMSNETS), Bengaluru, India, 7-11 January 2020. doi:10.1109/comsnets48256.2020.9027313

Li, H., Zhu, L., Shen, M., Gao, F., Tao, X., and Liu, S. (2018). Blockchain-Based Data Preservation System for Medical Data. J. Med. Syst. 42, 141. doi:10.1007/ s10916-018-0997-3

Lv, Z., and Piccialli, F. (2021). The Security of Medical Data on Internet Based on Differential Privacy Technology. ACM Trans. Internet Technol. 21, 1-18. doi:10.1145/3382769

Maleshkova, M., Pedrinaci, C., and Domingue, J. (2010). "Investigating Web APIs on the World Wide Web," in Web Services (ECOWS)," in 2010 IEEE 8th European Conference on (IEEE), Ayia Napa, Cyprus, 1-3 December 2010, 107-114.

Musamih, A., Salah, K., Jayaraman, R., Arshad, J., Debe, M., Al-Hammadi, Y., et al. (2021). A Blockchain-Based Approach for Drug Traceability in Healthcare Supply Chain. IEEE Access 9, 9728-9743. doi:10.1109/ ACCESS.2021.3049920

Narikimilli, N. R. S., Kumar, A., Antu, A. D., and Xie, B. (2020). "Blockchain Applications in Healthcare - A Review and Future Perspective," in Blockchain-ICBC 2020. Editors Z. Chen, L. Cui, B. Palanisamy, and L.-J. Zhang (Cham: Springer International Publishing), 198-218. doi:10.1007/978-3-030-59638-5_14

Omar, I. A., Jayaraman, R., Debe, M. S., Salah, K., Yaqoob, I., and Omar, M. (2021). Automating Procurement Contracts in the Healthcare Supply Chain Using Blockchain Smart Contracts. IEEE Access 9, 37397-37409. doi:10.1109/ACCESS.2021.3062471

Pilkington, M. (2016). "Blockchain Technology: Principles and Applications," in Research Handbook on Digital Transformations (Northampton, USA: Edward Elgar Publishing).

Protocol Labs Inc (2021). IPFS powers the Distributed Web. Available at: https:// ipfs.io/ (Accessed May 22, 2021).

Qiu, J., Liang, X., Shetty, S., and Bowden, D. (2018). “Towards Secure and Smart Healthcare in Smart Cities Using Blockchain,” in 2018 IEEE International Smart Cities Conference (ISC2), Kansas City, USA, 16-19 September 2018, 1-4.

Riso, B., Tupasela, A., Vears, D. F., Felzmann, H., Cockbain, J., Loi, M., et al (2017). Ethical Sharing of Health Data in Online Platforms - Which Values Should Be Considered? Life Sci. Soc. Pol. 13, 12. doi:10.1186/ s40504-017-0060-z

Tith, D., Lee, J.-S., Suzuki, H., Wijesundara, W. M. A. B., Taira, N., Obi, T., et al. (2020). Patient Consent Management by a Purpose-Based Consent Model for Electronic Health Record Based on Blockchain 
Technology. Healthc. Inform. Res. 26, 265-273. doi:10.4258/ hir.2020.26.4.265

Usman, M., and Qamar, U. (2020). Secure Electronic Medical Records Storage and Sharing Using Blockchain Technology. Proced. Comput. Sci. 174, 321-327. doi:10.1016/j.procs.2020.06.093

Vyas, J. D., Han, M., Li, L., Pouriyeh, S., and He, J. S. (2020). "Integrating Blockchain Technology into Healthcare,", in Proceedings of the 2020 ACM Southeast Conference. Editors M. Chang, D. Lo, and E. Gamess (New York, NY, USA: ACM), 197-203. doi:10.1145/3374135.3385280

Wu, H., Dwivedi, A. D., and Srivastava, G. (2021). Security and Privacy of Patient Information in Medical Systems Based on Blockchain Technology. ACM Trans. Multimedia Comput. Commun. Appl. 17, 1-17. doi:10.1145/ 3408321

Zhou, L., Wang, L., and Sun, Y. (2018). MIStore: a Blockchain-Based Medical Insurance Storage System. J. Med. Syst. 42, 149. doi:10.1007/s10916-0180996-4
Conflict of Interest: The authors declare that the research was conducted in the absence of any commercial or financial relationships that could be construed as a potential conflict of interest.

Publisher's Note: All claims expressed in this article are solely those of the authors and do not necessarily represent those of their affiliated organizations, or those of the publisher, the editors and the reviewers. Any product that may be evaluated in this article, or claim that may be made by its manufacturer, is not guaranteed or endorsed by the publisher.

Copyright (c) 2021 Westphal and Seitz. This is an open-access article distributed under the terms of the Creative Commons Attribution License (CC BY). The use, distribution or reproduction in other forums is permitted, provided the original author(s) and the copyright owner(s) are credited and that the original publication in this journal is cited, in accordance with accepted academic practice. No use, distribution or reproduction is permitted which does not comply with these terms. 